\title{
The Galactic Disk Distribution of Dust Emission Features in Planetary Nebulae
}

\author{
S. Casassus and P.F. Roche
}

Astrophysics, Oxford University, Keble Road, Oxford OX1 3RH, UK

\begin{abstract}
The properties of the carbon and oxygen chemical balance in planetary nebulae (PNe) are analysed through mid infrared spectroscopy of warm dust emission features in a sample of 72 objects. The adoption of a statistical distance scale shows that the galactic disk distribution of warm dust types in $\mathrm{PNe}$ is rather homogeneous with height over the galactic plane, and that there is a trend for the proportion of $\mathrm{PNe}$ with $\mathrm{O}$ rich dust signatures to decrease with galactocentric radius. Models of the galactic distribution of $\mathrm{PNe}$ compositions require a minimum progenitor mass of $1.2 \mathrm{M}_{\odot}$, although the observational constraints suffer from the smallness of the sample. This initial investigation is however an incentive to pursue the use of warm dust emission in PNe to study their progenitor population in various galactic environments.
\end{abstract}

\section{Introduction}

In the context of single star evolution, planetary nebulae (PNe) compositions reflect the processing undergone by the progenitor stars in their evolution up the Asymptotic Giant Branch. The dredge up of carbon synthesised in the He shell flashes alters the chemical balance in the envelope. But if the temperature at the bottom of the convective envelope is high enough, CNO processing enhances ${ }^{13} \mathrm{C}$ and ${ }^{14} \mathrm{~N}$ at the expenses of ${ }^{12} \mathrm{C}$ and ${ }^{16} \mathrm{O}$, and prevents dredged up carbon from reaching the surface. This process, often referred to as 'hot bottom burning' (HBB), produces radical changes in the predicted surface compositions. Thus a sequence is expected in nebular $\mathrm{C} / \mathrm{O}$ ratio which initially increases with main sequence mass, but then decreases again as HBB occurs. Another consequence of HBB is a strong enhancement of the nitrogen surface abundance; HBB is the only mechanism for single star evolution that can explain the highest $\mathrm{N} / \mathrm{O}$ ratios in $\mathrm{PNe}$.

The $\mathrm{C} / \mathrm{O}$ chemical balance in $\mathrm{PNe}$ can be determined through gas phase abundances, but also through the warm dust emission signatures at $10 \mu \mathrm{m}$. The dust signatures fall into 4 groups (see Roche 1987), and correspond to silicate grains, silicon carbide ( $\mathrm{SiC}$ ), the unidentified infrared bands (UIR), or result in either weak or unclassified continuum emission. The link with the carbon to oxygen ratio in the nebulae has been established by comparing with the gas phase abundances (e.g. Barlow 1983, Roche 1989). Silicate dust bands indicate an $\mathrm{O}$ rich environment, $\mathrm{SiC}$ a $\mathrm{C}$ rich environment, and UIR bands correlate with a strong overabundance of $\mathrm{C}$ relative to $\mathrm{O}$. 
We used the dust signatures in a sample of $72 \mathrm{PNe}$ as a systematic indicator of the $\mathrm{C} / \mathrm{O}$ chemical balance, and obtained their galactic disk distribution (Sect. 2.). The selection criteria were that the nebulae be compact, less than 10 " in diameter, and that their IRAS $12 \mu \mathrm{m}$ flux be higher than $0.5 \mathrm{Jy}$. The distribution of dust emission types is rather homogeneous with height above the galactic plane, and there seems to be a trend for a decreasing fraction of silicate emission PNe towards the outer Galaxy. We also confirmed, through crude galactic disk and synthetic AGB models, that the properties of the distribution can be understood in terms of single star evolution (Sect. 3.).

Although the warm dust emission types are likely to be affected by phenomena other than AGB evolution (e.g. interacting binaries), these results support the statistical interpretation of warm dust emission types in samples of $\mathrm{PNe}$ to investigate the progenitor populations.

\section{Warm dust emission features in a sample of compact PNe}

Figure 1a is a comparison between PNe types (Peimbert 1978) and dust signatures, for the PNe in our sample with known nitrogen abundance (with references in Casassus \& Roche 1998). PNe of type II are sub-divided according to the classification described in Faúndez-Abans \& Maciel (1987). The nitrogen rich $\mathrm{PNe}$ are the type I PNe, with $\log (\mathrm{N} / \mathrm{O})>-0.3$, and the type IIa PNe which have $\log (\mathrm{N} / \mathrm{O})>-0.6$. PNe with no nitrogen enrichment are the type IIb PNe, with Vlsr deviating from galactic disk circular motion by less than $60 \mathrm{~km} \mathrm{~s}^{-1}$, or else the type III PNe. The sequence in Peimbert types is indicative of progenitor mass, the highest being associated with type I. One would therefore expect a stratification in height above the galactic plane as a function of Peimbert type, as in Maciel \& Dutra (1992). Such a stratification is indeed present in our sample, as is illustrated in Fig. 1b, based on the distance scale by Zhang (1995). Inside the solar circle, the root mean square height over the plane, $z_{\mathrm{rms}}$, is $0.14 \mathrm{kpc}$ for type I, 0.37 for type IIa, 0.48 for type IIb, and 0.67 for type III. This stratification is also an indication that our sample is big enough for a statistical study. Out of $50 \mathrm{PNe}$ in our sample with known $\mathrm{N} / \mathrm{O}$ ratios, $30 \pm 5 \%$ are type I, which is typical of PNe catalogues (e.g. Maciel \& Dutra 1992), and we seem to sample homogeneously the disk PNe population.

Thus, UIR emission is related to higher progenitor masses, silicate emission is found mainly for low mass progenitors, but also for the most massive ones. $\mathrm{SiC}$ emission is related to intermediate nitrogen abundances, whereas 'weak' continuum $\mathrm{PNe}$ are equally represented.

Fig. 2 shows the spatial distribution of dust emission types, with distances on the scale by Zhang (1995). We list the properties of the dust type distributions in Table 1, where $\mathrm{N}$ stands for number, and the total number of sources was Ntot $=53,33$ for $\mathrm{R}<\mathrm{Ro}$, and 20 for $\mathrm{R}>\mathrm{Ro}$. Although there is a concentration of UIR emission PNe at low latitudes, the vertical distribution of $\mathrm{PNe}$ is rather homogeneous when compared to the Peimbert types, with $\mathrm{z}_{\mathrm{rms}}=0.45 \pm .1 \mathrm{kpc}$ for $\mathrm{O}$ rich $\mathrm{PNe}$, and $\mathrm{z}_{\mathrm{rms}}=0.35 \pm .04 \mathrm{kpc}$ for $\mathrm{C}$ rich PNe. There seems to be a trend towards a decreasing relative proportion of silicate dust type nebulae from $27 \%$ in the inner Galaxy, to $15 \%$ outside the solar circle, but as the standard error is $\sim 10 \%$ this decrease corresponds to only one sigma. 

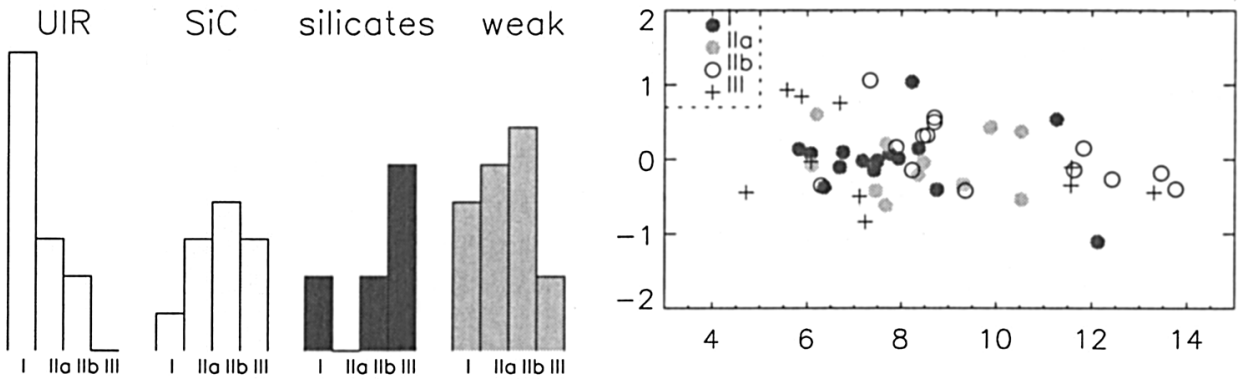

Figure 1. The relationships between the dust signatures and progenitor mass. Left hand plot: the number of $\mathrm{PNe}$ of each dust emission types as a function of Peimbert types. Right hand plot: the vertical distribution of $\mathrm{PNe}$ types, with $\mathrm{R}$ (galactocentric radius) in $\mathrm{x}$, and $\mathrm{z}$ (height over the plane) in $\mathrm{y}$.

Table 1. The properties of the distribution of planetary nebulae dust types.

\begin{tabular}{rccccc}
\hline & $b_{\text {rms }}$ & $\mathrm{z}_{\text {rms }}$ & \multicolumn{2}{c}{$\mathrm{N} /$ Ntot } & \\
type & {$[$ degrees $]$} & {$[100 \mathrm{pc}]$} & & $\mathrm{R}<\mathrm{Ro}$ & $\mathrm{R}>\mathrm{Ro}$ \\
\hline Silicates & $\mathbf{8 . 0}$ & $\mathbf{4 . 5}$ & $\mathbf{0 . 2 3}$ & 0.27 & 0.15 \\
SiC & $\mathbf{7 . 9}$ & $\mathbf{3 . 8}$ & $\mathbf{0 . 3 0}$ & 0.21 & 0.45 \\
UIR & $\mathbf{4 . 2}$ & $\mathbf{3 . 0}$ & $\mathbf{0 . 4 7}$ & 0.52 & 0.40 \\
\hline
\end{tabular}
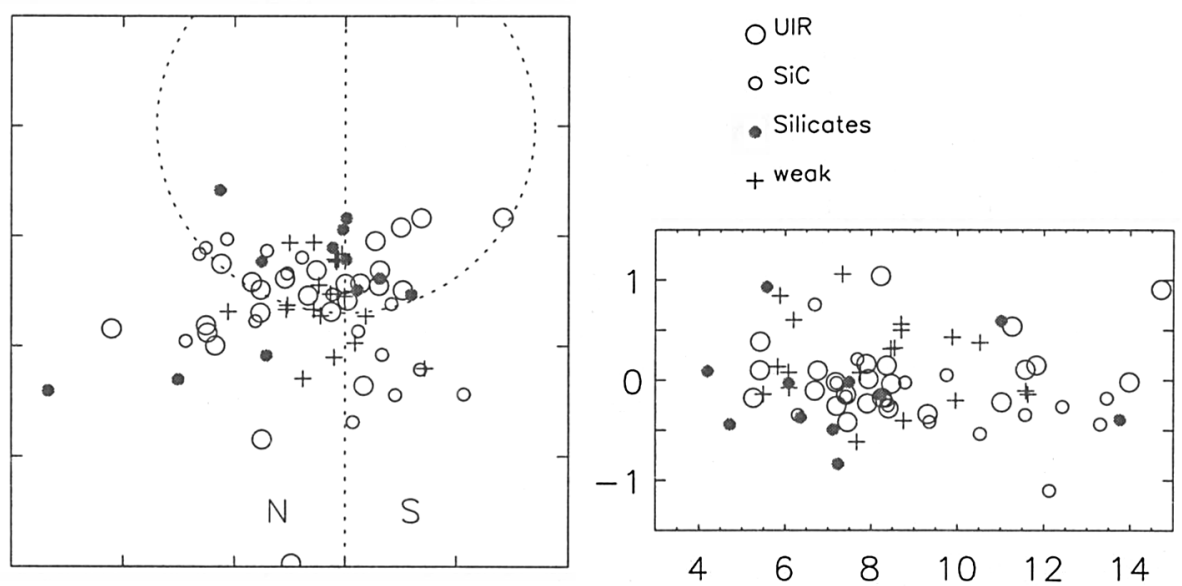

Figure 2. The galactic disk distribution of dust emission types in PNe. Left: face on view of the disk (ticks every $5 \mathrm{kpc}$ ). Right: vertical distribution, in $\mathrm{kpc}$. 
It was mentioned that $\mathrm{PNe}$ with no warm dust are equally distributed among Peimbert types. They also have high $6 \mathrm{~cm}$ fluxes, which together with the selection criteria of compact angular size, explains why they are on average closer. PNe with weak continuum probably correspond to later evolutionary stages. Their gas phase $\mathrm{C} / \mathrm{O}$ ratios reflect the proportions for the whole sample, with an average at 1.1 and a dispersion of $1.2 \mathrm{FWHM}$, again supporting that these are more evolved $\mathrm{PNe}$ (as opposed to $\mathrm{PNe}$ with $\mathrm{C} / \mathrm{O} \sim 1$ ).

Although all known IR bright and compact $\mathrm{PNe}$ were included in this study, further observations are required to improve the statistics.

\section{Synthetic distribution}

We constructed a synthetic AGB model that should be considered as an observational requirement only. The model will be fully described elsewhere (Casassus \& Roche 1998), but it follows the prescriptions compiled by Groenewegen \& de Jong (1993). Our aim was to check whether AGB evolution can explain the trends in the chemical composition of PNe. But we noted that existing AGB models cannot account for the continuous range of nitrogen abundances observed in LMC PNe (Leisy \& Dennefeld 1996), and the fact that $60 \%$ of the type I PNe in our sample have $\mathrm{C}$ rich dust (confirmed by the gas phase abundances). We resolved this discrepancy by implementing $\mathrm{HBB}$ through a simple $\mathrm{CNO}$ tri-cycle at temperature $T_{B}$, with a typical exposure time $t_{H B B}$ and cumulative exposures every $\tau_{\text {conv }}=0.5$ yrs ( $\sim$ convective turnover timescale). We fixed $t_{\mathrm{HBB}}=10^{-5} \mathrm{yrs}$ so as to obtain ${ }^{12} \mathrm{C} /{ }^{13} \mathrm{C}$ ratios at $\mathrm{CN}$ equilibrium values for the most massive stars. We account for $\mathrm{N}$ enrichment in $\mathrm{C}$ rich $\mathrm{PNe}$ by a linear dependence of $\mathrm{T}_{\mathrm{B}}$ with core mass, $\mathrm{dT}_{\mathrm{B}} / \mathrm{dM}_{c}=2 \times 10^{9} \mathrm{KM}_{\odot}^{-1}$. An increase of $\mathrm{T}_{\mathrm{B}}$ on the AGB strong enough to produce $\mathrm{C}$ and $\mathrm{N}$ rich $\mathrm{PNe}$ was obtained by Renzini \& Voli (1981), although their mass loss rate was underestimated. The model was also required to reproduce the luminosity function of C stars in the LMC (Cohen et al. 1981) and the initial-final mass relation (Weidemann \& Koester 1983; Weidemann 1987), which fixed the minimum core mass for dredge-up $M_{c}^{m i n}=0.58 \mathrm{M}_{\odot}$, the dredge-up parameter $\lambda=0.6$, and mass loss parameter $\eta=5$ (Reimers law). The resulting PNe abundances, averaged over the last 25000 yrs of AGB evolution, are shown in Fig. 3.

We also needed a crude galactic disk model, including as much as possible observed distributions rather than model results. The star formation rate as a function of galactocentric radius was extrapolated from the UCHII region survey in Bronfman et al. (1998). We used the results presented in Köppen \& Cuisinier (1997) for the age of each star and the treatment of radial diffusion. For the vertical structure of the disk, we used the approximations in Talbot \& Arnett (1975) and the age-velocity relation by Wielen (1977). As in Carraro et al. (1998), we took a radial metallicity gradient of $-0.07 \mathrm{dex} / \mathrm{kpc}$ constant in time. $\mathrm{Z}(\mathrm{R}=\mathrm{Ro}, \mathrm{t})$ was required to match the age metallicity relation by Meusinger et al. (1991). The model is therefore a very simple one, but it should be kept in mind that we are modelling a PNe distribution based on a statistical distance scale. This distribution cannot be regarded as absolute, only the relative distributions of the different PNe types contain valuable information. 

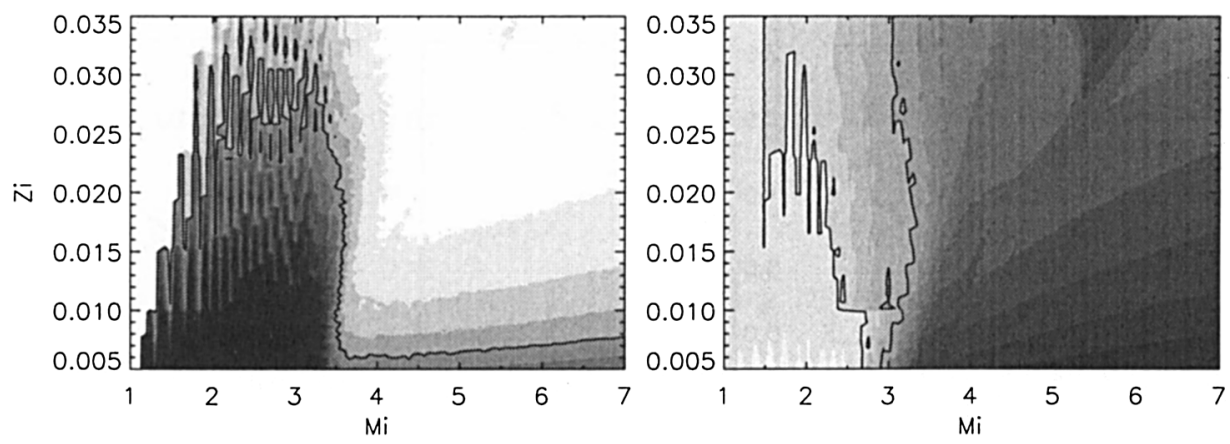

Figure 3. PNe abundances as a function of initial mass in $\mathrm{x}$, initial metallicity in $\mathrm{y}$. The left hand plot shows the $\mathrm{C} / \mathrm{O}$ ratio in grey scale, with darker contours at $.2, .4, .6, \ldots$ and the solid line is at $\mathrm{C} / \mathrm{O}=1$. The right hand plot shows $\log (\mathrm{N} / \mathrm{O})$, with darker grey scale contours at $-.9,-.8, \ldots, 1$ and the solid lines represent the contours for type IIa and type I PNe.

In order to link the modelled and observed PNe galactic distributions, we averaged the fraction of flux emitted in the IRAS $12 \mu \mathrm{m}$ band, and applied our flux selection criterion to the synthetic population. But many selection effects come from the $\mathrm{PNe}$ catalogues themselves, and we adopted a minimum galactocentric radius of 0.5 Ro (as can be inferred from Fig. 2, see also Durand et al. 1998). Since PNe with no warm dust correspond to later evolutionary stages, the link between the computed $\mathrm{C} / \mathrm{O}$ ratios and the dust features is very tight. Silicates represent $\mathrm{C} / \mathrm{O}<1$, and $\mathrm{SiC}$ and UIR bands $\mathrm{C} / \mathrm{O}>1$.

The properties of the synthetic distribution that we would like to compare to the observations are shown in Fig. 4. PNe compositions were computed by averaging the stellar mass loss over the last 2000 years of evolution on the AGB to compare with the dust signatures, and over the last 25000 years to compare with the gas phase abundances. The minimum mass for the progenitors of compact and infrared bright $\mathrm{PNe}, \mathrm{M}^{\mathrm{min}}$, was kept as a free parameter. Fig. 4a and $4 \mathrm{~b}$ are the results from $t_{P N}=2000 \mathrm{yrs}$; Fig. $4 \mathrm{a}$ shows the fraction of $\mathrm{O}$ rich $\mathrm{PNe}$ and Fig. $4 \mathrm{~b}$ is the ratio of the root mean square height over the plane for $\mathrm{O}$ rich and $\mathrm{C}$ rich $\mathrm{PNe}$. Fig. 4c shows the proportion of $\mathrm{N}$ rich $\mathrm{PNe}$, as obtained with $t_{P N}=25000$ yrs. Values of $\mathrm{M}^{\text {min }}$ between $1.15 \mathrm{M}_{\odot}$ and $1.35 \mathrm{M}_{\odot}$ can account for all the observed properties of $\mathrm{PNe}$ within their standard errors. With $\mathrm{M}^{\mathrm{min}}=1.2 \mathrm{M}_{\odot}$, the fraction of type I PNe with $\mathrm{C}$ rich dust signatures is $70 \%$, whereas the fraction with gas phase $\mathrm{C} / \mathrm{O}>1$ is $40 \%$.

\section{Conclusions}

We undertook a systematic study of the $\mathrm{C} / \mathrm{O}$ chemical balance at the end of the AGB through warm dust emission in a sample of 72 compact and infrared bright PNe. The properties of the galactic disk distribution of the dust signatures in $\mathrm{PNe}$ are consistent with a simple AGB model, and require a minimum progenitor mass of about $1.2 \mathrm{M}_{\odot}$ and an ejection age of 2000 years. These results are 

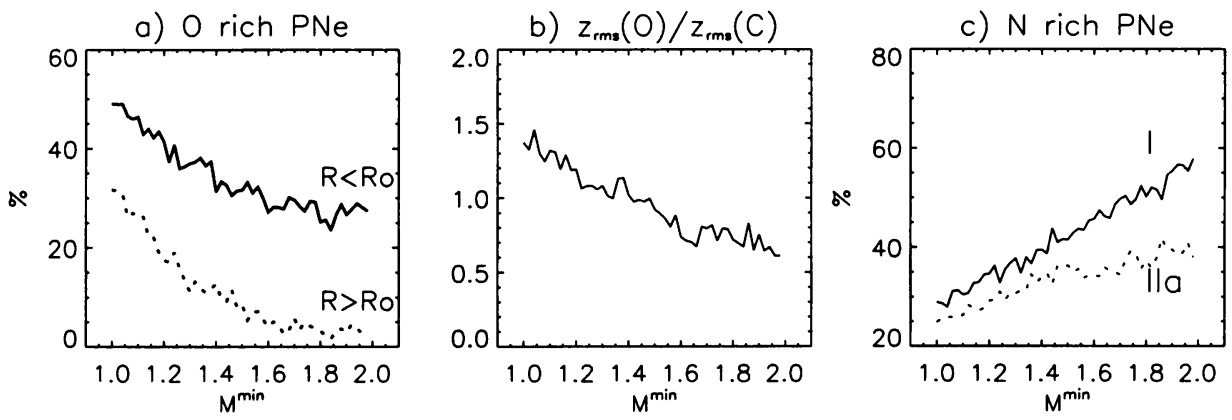

Figure 4. Properties of the synthetic PNe population as a function of minimum main sequence progenitor mass, $\mathrm{M}^{\text {min }}$.

an incentive to pursue the use of warm dust emission in PNe to study their progenitor population in various galactic environments.

\section{References}

Barlow B.M., 1983, in "Planetary Nebulae", IAU Symposium 103, ed. D.R. Flower, p. 105

Bronfman L., Casassus S., May J., Nyman L.-Å., 1998, in preparation

Carraro G., Ng Y.K., Portinari L., 1998, MNRAS 296, 1045

Casassus S., Roche P.F., 1998, in preparation

Cohen J.G., Frogel J.A, Persson S.E., Elias J.H., 1981, ApJ 249, 481

Durand S., Acker A., Zijlstra A., 1998, A\&A 132, 13

Faúndez-Abans M., Maciel W.J., 1987, A\&A 183, 324

Groenewegen M.A.T., de Jong T., 1993, A\&A 267, 410

Köppen J., Cuisinier F., 1997, A\&A 319, 98

Leisy P., Dennefeld M., 1996, A\&AS 116, 95

Maciel W.J., Dutra C.M., 1992, A\&A 262, 271

Meusinger H., Reimann H.-G., Stecklum B., 1991, A\&A 245, 57

Peimbert M., 1978, in "Planetary Nebulae", IAU Symposium 76, Reidel, p. 215

Roche P.F., 1987, in "Planetary and Proto-Planetary Nebulae: From IRAS to ISO", A. Priete Martinez (ed.), p. 45

Roche P.F., 1989, in "Planetary Nebulae", IAU Symposium 131, ed. S. Torres

Peimbert, p. 117

Renzini A., Voli M., 1981, A\&A 94, 175

Talbot R.J., Arnett W.D., 1975, ApJ 197, 551

Weidemann V., 1987, A\&A 188, 74

Weidemann V., Koester D., 1983, A\&A 121, 77

Wielen R., 1977, A\&A 60, 263

Zhang C.Y., 1995, ApJS 98, 659 